\title{
Droplet Deformation Prediction With the Droplet Deformation and Breakup Model (DDB)
}

Mario Vargas

Glenn Research Center, Cleveland, Ohio 


\section{NASA STI Program . . . in Profile}

Since its founding, NASA has been dedicated to the advancement of aeronautics and space science. The NASA Scientific and Technical Information (STI) program plays a key part in helping NASA maintain this important role.

The NASA STI Program operates under the auspices of the Agency Chief Information Officer. It collects, organizes, provides for archiving, and disseminates NASA's STI. The NASA STI program provides access to the NASA Aeronautics and Space Database and its public interface, the NASA Technical Reports Server, thus providing one of the largest collections of aeronautical and space science STI in the world. Results are published in both non-NASA channels and by NASA in the NASA STI Report Series, which includes the following report types:

- TECHNICAL PUBLICATION. Reports of completed research or a major significant phase of research that present the results of NASA programs and include extensive data or theoretical analysis. Includes compilations of significant scientific and technical data and information deemed to be of continuing reference value. NASA counterpart of peer-reviewed formal professional papers but has less stringent limitations on manuscript length and extent of graphic presentations.

- TECHNICAL MEMORANDUM. Scientific and technical findings that are preliminary or of specialized interest, e.g., quick release reports, working papers, and bibliographies that contain minimal annotation. Does not contain extensive analysis.

- CONTRACTOR REPORT. Scientific and technical findings by NASA-sponsored contractors and grantees.
- CONFERENCE PUBLICATION. Collected papers from scientific and technical conferences, symposia, seminars, or other meetings sponsored or cosponsored by NASA.

- SPECIAL PUBLICATION. Scientific, technical, or historical information from NASA programs, projects, and missions, often concerned with subjects having substantial public interest.

- TECHNICAL TRANSLATION. Englishlanguage translations of foreign scientific and technical material pertinent to NASA's mission.

Specialized services also include creating custom thesauri, building customized databases, organizing and publishing research results.

For more information about the NASA STI program, see the following:

- Access the NASA STI program home page at http://www.sti.nasa.gov

- E-mail your question to help@sti.nasa.gov

- Fax your question to the NASA STI Information Desk at 443-757-5803

- Phone the NASA STI Information Desk at 443-757-5802

- Write to: STI Information Desk NASA Center for AeroSpace Information 7115 Standard Drive Hanover, MD 21076-1320 


\section{Droplet Deformation Prediction With the Droplet Deformation and Breakup Model (DDB)}

Mario Vargas

Glenn Research Center, Cleveland, Ohio

Prepared for the

Atmospheric Space Environments Conference

sponsored by the American Institute of Aeronautics and Astronautics

New Orleans, Louisiana, June 25-28, 2012

National Aeronautics and

Space Administration

Glenn Research Center

Cleveland, Ohio 44135 


\section{Acknowledgments}

Thanks to Dr. Andy Broeren and Dr. Jen Chin Tsao for their patience and encouragement to the author to finish the paper. The present work augments the experimental work performed in an international agreement between NASA and the Instituto Nacional de Técnica Aeroespacial (INTA). It is funded by the Atmospheric Environment Safety Technologies (AEST) Project, Aviation Safety Program (AvSP). Especial thanks to Dr. Ron Colantonio and Ms. Mary Wadel for their support of the effort.

Trade names and trademarks are used in this report for identification only. Their usage does not constitute an official endorsement, either expressed or implied, by the National Aeronautics and Space Administration.

Level of Review: This material has been technically reviewed by technical management.

Available from

NASA Center for Aerospace Information 7115 Standard Drive

Hanover, MD 21076-1320
National Technical Information Service 5301 Shawnee Road Alexandria, VA 22312 


\title{
Droplet Deformation Prediction With the Droplet Deformation and Breakup Model (DDB)
}

\author{
Mario Vargas \\ National Aeronautics and Space Administration \\ Glenn Research Center \\ Brook Park, Ohio 44135
}

\begin{abstract}
The Droplet Deformation and Breakup Model was used to predict deformation of droplets approaching the leading edge stagnation line of an airfoil. The quasi-steady model was solved for each position along the droplet path. A program was developed to solve the non-linear, second order, ordinary differential equation that governs the model. A fourth order Runge-Kutta method was used to solve the equation. Experimental slip velocities from droplet breakup studies were used as input to the model which required slip velocity along the particle path. The center of mass displacement predictions were compared to the experimental measurements from the droplet breakup studies for droplets with radii in the range of 200 to $700 \mu \mathrm{m}$ approaching the airfoil at 50 and $90 \mathrm{~m} / \mathrm{sec}$. The model predictions were good for the displacement of the center of mass for small and medium sized droplets. For larger droplets the model predictions did not agree with the experimental results.
\end{abstract}

\section{Nomenclature}

$\begin{array}{ll}A_{s} & \text { Area of the deforming droplet } \\ a & \text { major semi-axis of oblate spheroid } \\ b & \text { minor semi-axis of oblate spheroid } \\ \text { Bo } & \text { Bond number } \\ \text { Cd } & \text { Drag coefficient } \\ \text { DDB } & \text { Droplet Deformation and Breakup Model } \\ R & \text { Droplet radius } \\ \text { Re } & \text { Reynolds number } \\ \text { SLD } & \text { Supercooled Large Droplets } \\ \text { TAB } & \text { Taylor Analogy Break-up Model } \\ U_{\text {rel }} & \text { Slip Velocity, relative velocity between the droplet and the air } \\ \text { We } & \text { Weber number }\end{array}$

\section{Introduction}

Supercooled Large Droplet (SLD) breakup near the leading edge of large transport airfoils may alter the mass flux of water reaching the leading edge and be a factor that needs to be accounted for in the prediction of ice accretion formation in icing codes. The present research is part of current efforts to understand, measure and predict SLD breakup near the leading edge of large transport wings. The studies will help determine if icing codes need to be modified to account for droplet breakup when the size of the droplets is in the SLD regime.

Recent experimental studies (Refs. 1 to 3) of Supercooled Large Droplet (SLD) deformation and breakup near the leading edge of airfoils have provided imaging data and measurements of important parameters along the droplet paths. The experimental data from these studies can be used to evaluate 
previously developed droplet breakup models (Refs. 4 to 7) and determine the limits of their applicability to the experimental configuration where droplets deform and break up near the leading edge of an airfoil.

When there is a difference between the velocity of a droplet and the velocity of the air, called slip velocity, a flow field develops around the droplet and aerodynamic forces begin to deform it. As the viscous forces and pressure forces deform the droplet, its shape changes which, in turn modifies the flow field and the aerodynamic forces acting on the droplet. The flow field and the droplet deformation are coupled physical processes. In a strict sense the modeling of droplet deformation requires the mathematical equations describing the coupling between the two processes. This is a very complex situation because the droplet is a liquid elastic body held together by surface tension, and internal circulation of water inside the droplet may be present. General models of droplet deformation do not capture all of the physics but use simplifications to make the solution more tractable. These models are valuable because they capture the important physical processes and provide insight into the phenomena. The only way to know if a model may be used in a given experimental configuration is to test it against the experimental results and find the limits of its usefulness and applicability.

Droplet breakup has been studied extensively in the past for experimental configurations (Refs. 4 to 7) where the droplet is subjected to a rapidly changing slip velocity. Current droplet breakup models were developed primarily for that type of configuration. For droplets which approach the airfoil along the stagnation line the droplets are subject to a gradual increase in the slip velocity that allows them to adjust their shape along the path and tolerate higher Weber numbers before beginning the actual breakup sequence. It is important to test the applicability of current droplet breakup models to this configuration because the models may help interpret experimental data, provide insights into the physics involved in the process and offer guidance in the planning of future experiments.

The Droplet Deformation and Breakup (DDB) Model and the Taylor Analogy Break-up (TAB) Model more closely model the kind of deformation observed in droplet breakup studies near the leading edge of airfoils. The DDB was selected for the current study to predict the droplet deformation along the path of the droplet. A program was developed to solve the second order, non-linear ordinary differential equation that governs the model. A fourth order Runge-Kutta method was used in the numerical scheme to solve the equation. Experimental values of the slip velocity were used as input to the model. The predicted displacements of the droplet center of mass were compared to the experimental measurements from current droplet breakup studies on a DBKUP airfoil geometry. Comparisons were generated for airfoil speeds of 50 and $90 \mathrm{~m} / \mathrm{sec}$ and droplet sizes from 200 to $700 \mu \mathrm{m}$.

\section{Background on Droplet Deformation Breakup Model}

The DDB model was proposed in 1993 by Ibrahim, Yang and Przekwas (Ref. 6). The model assumes that "the liquid droplet is deformed from an initial spherical shape of radius $R$ into an oblate spheroid of an ellipsoidal cross section with major semi-axis $a$ and minor semi-axis $b$ ". This assumption makes the model attractive for droplet deformation prediction near the leading edge of an airfoil because during experimental studies the droplets were observed to deform from a spherical shape to an oblate spheroid. For this reason the model was tested against the experimental data to determine its applicability.

The DDB model tracks the vertical motion of the center of mass of a half droplet (Fig. 1). It is based on conservation of mass and energy for half the droplet as it deforms. In the rest of this section, the derivation of the fundamental equation representing the model is presented, following Ibrahim's original derivation (Ref. 6).

Assuming the droplet does not exchange heat with its surroundings and that the only forces involved in the deformation of the droplet are the viscous forces, the pressure forces and the surface tension forces, the energy equation for the half-droplet is:

$$
\frac{d E}{d t}=-\frac{d W}{d t}
$$


where $E$ is the energy and $W$ is the work done on the droplet by the pressure and viscous forces.

Therefore

$$
\frac{d E_{\text {kinetic }}}{d t}+\frac{d E_{\text {potential }}}{d t}=\frac{d W_{\text {pressure }}}{d t}+\Phi
$$

where $\Phi$ is the viscous dissipation. Each term in Equation (2) will be evaluated next.

Kinetic Energy:

$$
\frac{d E_{\text {kinetic }}}{d t}=m \cdot\left(\frac{d v}{d t}\right) \cdot v=\frac{2}{3} \pi \cdot R^{3} \cdot \rho_{l} \cdot v \cdot \frac{d v}{d t}=\frac{2}{3} \pi \cdot R^{3} \cdot \rho_{l} \cdot \frac{d y}{d t}\left(\frac{d^{2} y}{d t^{2}}\right)
$$

where $m$ is the mass of half the droplet, $\rho_{l}$ the density of water, $R$ the radius of the droplet, $v=d y / d t$ and $d v / d t=d^{2} y / d t^{2}$ are the velocity and acceleration respectively of the center of mass of the deforming half droplet (Fig. 1).

Potential Energy

$$
\frac{d E_{\text {potential }}}{d t}=\frac{1}{2} \sigma \cdot\left(\frac{d A_{s}}{d t}\right)
$$

where $\sigma$ is the surface tension (energy/unit area), $1 / 2$ is the factor for the half droplet energy balance, $A_{s}$ is the area of the deforming droplet. The area of the deforming droplet is the area of the oblate spheroid with major semi-axis $a$ and minor semi-axis $b$. It is given by:

$$
A_{s}=2 \pi a^{2}+2 \pi b^{2} \cdot \phi
$$

where

$$
\phi=\frac{1}{2 \varepsilon} \cdot \ln \left(\frac{1+\varepsilon}{1-\varepsilon}\right)
$$

and

$$
\varepsilon=\sqrt{1-\left(\frac{a}{b}\right)^{-2}}
$$

In the model $A_{s}$ is approximated by assuming $\phi=1$ :

$$
A_{s}=2 \pi\left(a^{2}+b^{2}\right)
$$

For increasing values of $a / b, \phi$ quickly increases from a value of 1 , but the approximation is reasonable within the overall calculation of $A_{s}$. As the droplet deforms into an oblate spheroid, the value of the major semi-axis $a$ increases while the value of the minor semi-axis $b$ decreases. The first term in the Equation (5) becomes larger, the second term smaller, and the impact of the error in the calculation of the second term is not as large for the calculation of $A_{s}$. In Figure 2, the ratio of the approximation to the 
area divided by the exact value is plotted against the ratio $a / b$ for five different droplets. The ratio decreases to a minimum at $a / b=2$ and then increases.

Before discussing the derivative with respect to time of Equation (8), two other terms that will be used are introduced. From the conservation of volume:

$$
\frac{4}{3} a^{2} \cdot b=\frac{4}{3} \pi R^{3}
$$

therefore

$$
b=\frac{R^{3}}{a^{2}}
$$

The distance of the center of mass in terms of the major semi-axis $a$ was approximated by the distance of the center of mass for an area of a semi-ellipse of altitude $a$ :

$$
y=\frac{4}{3 \pi} a
$$

which can also be noted as

$$
a=c \cdot y
$$

where

$$
c=\frac{3 \pi}{4}
$$

For half an oblate spheroid the distance of the center of mass in terms of the major semi-axis $a$ is given by (3/8) $a$. The approximation given in Equation (11) introduces an error of 12 percent. The exact value of $c=8 / 3$ was used when running the model for this study.

Taking the derivative with respect to time of $A_{s}$ and substituting in Equation (8) the expressions for $b$ and $a$ from Equations (10) and (12) gives:

$$
\frac{d A_{s}}{d t}=\frac{9 \pi^{3}}{4} y\left[1-2 \cdot\left(\frac{c \cdot y}{R}\right)^{-6}\right] \cdot\left(\frac{d y}{d t}\right)
$$

Substituting Equation (14) into Equation (4) gives the expression for the potential energy:

$$
\frac{d E_{\text {potential }}}{d t}=\frac{9 \pi^{3}}{8} \sigma \cdot y\left[1-2 \cdot\left(\frac{c \cdot y}{R}\right)^{-6}\right] \cdot\left(\frac{d y}{d t}\right)
$$

The rate of change of the work done by the pressure is given by:

$$
\frac{d W_{\text {pressure }}}{d t}=-\frac{1}{2} \cdot p \cdot A_{p} \cdot\left(\frac{d y}{d t}\right)
$$


Where $p$ is the pressure and $A_{p}$ is the projected area of the droplet. The projected area of the deformed droplet is approximated by the original projected area of the spherical droplet:

$$
A_{p} \approx \pi \cdot R^{2}
$$

This assumption is very restrictive because it limits the applicability of the model to small droplet deformations. But since $A_{p}$ is the projected area of the oblate spheroid and is given by $\pi \cdot a^{2}$, where $a$ is the major semi-axis of the oblate spheroid, the model can be modified to avoid the approximation in Equation (17) by substituting $\pi \cdot a^{2}$ for $\pi \cdot R^{2}$.

The value of the average pressure exerted on the front and back of the half oblate spheroid is assumed to be equal to the stagnation pressure of the droplet:

$$
p=\frac{1}{2} \cdot \rho_{g} \cdot U^{2} r e l
$$

where $\rho_{g}$ is the air density and $U_{\text {rel }}$ is the slip velocity. The approximation in Equation (18) is reasonable because it results from a dimensional analysis which implies that inertia force that deform the droplet is proportional to the stagnation pressure (Ref. 1).

Substituting Equations (17) and (18) into Equation (16) gives the rate of work done by the pressure as:

$$
\frac{d W_{\text {pressure }}}{d t}=-\frac{\pi}{4} \cdot R^{2} \cdot \rho_{g} \cdot U_{\text {rel }}^{2}\left(\frac{d y}{d t}\right)
$$

The negative sign in front of term indicates work is being done on the droplet by the pressure forces, following the standard convention. The rate of work done by the viscous forces is given by the viscous dissipation function (Ref. 6):

$$
\frac{d W_{\text {viscous }}}{d t}=\Phi=\frac{8}{3} \pi \cdot R^{3} \cdot \mu_{l} \cdot\left(\frac{1}{y} \frac{d y}{d t}\right)^{2}
$$

where $\mu_{l}$ is the water viscosity and $R$ the radius of the droplet. Substitution of Equations (3), (15), (19), and (20) into Equation (2) gives the basic equation that governs the model:

$$
\begin{array}{r}
\frac{2}{3} \pi \cdot R^{3} \rho_{l}\left(\frac{d y}{d t}\right) \cdot\left(\frac{d^{2} y}{d t^{2}}\right)+\frac{9 \pi^{3}}{8} \sigma \cdot y\left[1-2 \cdot\left(\frac{c \cdot y}{R}\right)^{-6}\right] \cdot\left(\frac{d y}{d t}\right) \\
=\frac{\pi}{4} \cdot R^{2} \cdot \rho_{g} \cdot U_{r e l}^{2}\left(\frac{d y}{d t}\right)-\frac{8}{3} \pi \cdot R^{3} \cdot \mu_{l} \cdot\left(\frac{1}{y} \frac{d y}{d t}\right)^{2}
\end{array}
$$

To non-dimensionalize the equation the following dimensionless variables are defined:

$$
y^{*}=\frac{y}{R}
$$




$$
\begin{aligned}
& t^{*}=t \cdot\left(\frac{U_{r e l}}{R}\right) \\
& R e=\frac{\rho_{g} U_{r e l} R}{\mu_{g}}
\end{aligned}
$$

where $\rho_{g}$ is the density of the air and $\mu_{g}$ is the absolute viscosity of the air.

$$
W e=\frac{\rho_{g} U^{2} r e l R}{\sigma}
$$

where $\sigma$ is the surface tension of water.

$$
K=\frac{\rho_{l}}{\rho_{g}}
$$

Substituting Equations (22) to (26) into Equation (21) and re-arranging we get:

$$
K\left(\frac{d^{2} y^{*}}{d t^{* 2}}\right)+\frac{4 N}{R e} \frac{1}{y^{* 2}} \frac{d y^{*}}{d t^{*}}+\frac{27 \pi^{2}}{16 \cdot W e} y^{*}\left[1-2\left(c \cdot y^{*}\right)^{-6}\right]=\frac{3}{8}
$$

For readability, the asterisk is dropped from the $y$ and $t$ terms in Equation (27). The equation that represents the DDB model is now written:

$$
K\left(\frac{d^{2} y}{d t^{2}}\right)+\frac{4 N}{R e} \frac{1}{y^{* 2}} \frac{d y}{d t}+\frac{27 \pi^{2}}{16 \cdot W e} y\left[1-2(c \cdot y)^{-6}\right]=\frac{3}{8}
$$

This is a second order non-linear ordinary differential equation with two initial conditions given by:

$$
y=\frac{4}{3 \pi} \text { at } t=0
$$

where $y$ and $t$ are non-dimensional. This initial condition reflects the fact that the half-droplet is a hemisphere before any deformation begins. The second initial condition is:

$$
\frac{d y}{d t}=0 \text { at } t=0
$$

where the dimensionless initial velocity of the center of mass is assumed zero. Equations (28), (29), and (30) constitute the initial value problem. In addition to the water and air properties, the radius of the droplet $R$ and $U_{\text {rel }}$ are needed to solve Equation (28) together with its initial conditions. 


\section{Numerical Solution of the Initial Value Problem}

The initial value problem constituted by the ordinary differential equation and the boundary conditions of Equations (28), (29), and (30) was solved numerically. The first step in the numerical approach was to use a transformation of variables and convert the original problem into a system of two coupled first order ordinary differential equations with corresponding initial conditions.

$$
\begin{gathered}
K\left(\frac{d^{2} y}{d t^{2}}\right)+\frac{4 N}{R e} \frac{1}{y^{* 2}} \frac{d y}{d t}+\frac{27 \pi^{2}}{16 \cdot \mathrm{We}} y\left[1-2(c \cdot y)^{-6}\right]=\frac{3}{8} \\
y(0)=\frac{4}{3 \pi} \quad \frac{d y}{d t}(0)=0 \\
\frac{d y}{d z}=z \quad I C: y(0)=\frac{4}{3 \pi} \\
\frac{d z}{d t}=-\frac{4 N}{K \cdot \operatorname{Re}} \frac{1}{y^{2}} z-\frac{27 \pi^{2}}{16 K \cdot \mathrm{We}}\left[1-2 \cdot\left(\frac{3 \pi y}{4}\right)^{-6}\right]+\frac{3}{8 K}
\end{gathered}
$$

A fixed time step, fourth order Runge-Kutta (RK) integrator was used to solve the system in Equation (31). A second order RK was used to estimate the truncation error.

\section{Input Parameters Needed for the DDB Model and the Numerical Solution}

Two types of input parameters are required to test the DDB model: (1) The input parameters needed for the DDB model and (2) The input parameters needed for the numerical scheme to solve the initial value problem.

The input parameters for the DDB model are: density of air, viscosity of air, density of water, viscosity of water, surface tension of water, diameter of the droplet and the slip velocity.

The input parameters for the numerical solution of the initial value problem are: the number of first order differential equations, initial and final value of the independent variable in the interval where the solution is evaluated, number of steps in the interval, step size, and error tolerance.

\section{Experimental Data Used With the DDB Model}

Experimental studies of droplets approaching the leading edge of an airfoil along the stagnation line have provided data on droplet deformation and breakup (Refs. 1 to 3). The experiments were conducted in a rotating arm facility (Ref. 2). An airfoil was placed at the end of the arm and rotated at a given velocity. Water droplets from a TSI-100 droplet generator were allowed to fall at a specific location in the path of the airfoil being rotated. High speed movies of the droplet deformation and breakup were recorded. Digital image processing programs developed for the analysis of the data were used to track the droplets and measure the main parameters involved. Figure 3 is a sequence of images captured during these experiments at different times as a droplet approached the airfoil. Initially the droplet was spherical as there was no difference between the velocity of the droplet and the air (slip velocity). As the droplet 
approached the airfoil, the air velocity decreased and the droplet's slip velocity increased. The increase in the slip velocity generated aerodynamic forces that deformed the droplet initially to a symmetrical oblate spheroid (frame 40 in Fig. 3) and later to an asymmetrical oblate spheroid (frame 110 in Fig. 3), which is asymmetric with respect to the major semi-axis but rotationally symmetric with respect to the axis of motion. The image processing program measured the width and the height of the droplet along the path.

Figure 4 shows the variation in width of the droplet along its path. The droplet width decreased to a minimum and then increased. The location at which the width of a droplet is a minimum defines where droplet deformation ends and droplet breakup begins (Ref. 3). This corresponds to frame 192 in Figure 3. As the droplet width decreased, its height increased. Figure 5 shows the height of the droplet along its path. The height of the droplet is the major $a$ semi-axis (Fig. 1) of the oblate spheroid. The center of mass location is given by (3/8)a. The DDB model, which predicts center of mass of the deformed droplet, was only used to analyze the droplet deformation phase of the breakup and not the post droplet breakup phase. Data from five tracked droplets were used to test the DDB model. Table 1 presents the basic data for each droplet.

\section{Approach Taken and Main Assumption}

The approach taken to apply the DDB model to the deformation of the droplets was to assume that at each location along the trajectory the droplet is in quasi-steady equilibrium. Based on this assumption, the model was solved at each location along the path of the droplet. To solve the model equations at each location, the experimentally measured value of the slip velocity (Ref. 3) for that location was required as an input parameter for the model. The predictions of the displacement of the center of mass of the half droplet were compared to the experimentally measured values.

\section{Results and Discussion}

The DBB model, although highly nonlinear, behaved much like the classical spring-mass-friction case at a given location. The value of the center of mass oscillated around the final equilibrium value and as time increased it approached the equilibrium value (Fig. 6). The larger the experimental value of the slip velocity, the larger the amplitude of the initial oscillation, and the longer it took the system to reach equilibrium (Fig. 7).

The DDB model was applied to five cases of droplet deformation. In each case the experimentally measured value of the slip velocity was used as part of the input for the model. The model was run at each location where experimental data was available up until the onset of breakup. Figures comparing computational results to experiment are presented for each case. A discussion of each case follows.

Figure 8 shows the comparison between the model prediction and the experimental data for Case 1: Run 072211-1.8 droplet 4, droplet radius $199 \mu \mathrm{m}$, airfoil chord $0.710 \mathrm{~m}$ and airfoil velocity $50 \mathrm{~m} / \mathrm{sec}$. At this low velocity the small droplet deforms very little as it approaches the airfoil. This is reflected in the nearly constant value of the experimental measurement of the center of mass location. The model prediction agrees well with the experimental data. In general small droplets resist deformation more than larger ones because the small radius of curvature translates into a larger surface tension force.

Figure 9 shows the comparison between the model prediction and the experimental data for Case 2: Run 072611-18.6 droplet 1, droplet radius $287 \mu \mathrm{m}$, airfoil chord $0.710 \mathrm{~m}$ and airfoil velocity $90 \mathrm{~m} / \mathrm{sec}$. The droplet is also relatively small but the velocity of the airfoil is higher than for Case 1 resulting in a larger slip velocity. This larger slip velocity results in an increase in the droplet center of mass over Case 1 . The predictions for this case were also relatively good.

Figure 10 shows the comparison between the model prediction and the experimental data for Case 3: Run 072611-16.10 droplet 2, droplet radius $439 \mu \mathrm{m}$, airfoil chord $0.710 \mathrm{~m}$ and airfoil velocity $90 \mathrm{~m} / \mathrm{sec}$. The droplet is much larger than for Case 1 and 2. This results in a larger slip velocity and more 
deformation along the path. The model initially over predicts the value of the center of mass and then converges more closely to the experimental data starting at a distance of $-100 \mathrm{~mm}$ from the leading edge of the airfoil.

Figure 11 shows the comparison between the model prediction and the experimental data for Case 4: Run 072011-14B.1 droplet 1, droplet radius $516 \mu \mathrm{m}$, airfoil chord $0.710 \mathrm{~m}$ and airfoil velocity $90 \mathrm{~m} / \mathrm{sec}$. The droplet is somewhat larger than for Case 3. Initially the model over-predicts the position of the center of mass by 13 percent. The difference between the model and experiment then decreases until the droplet reaches a distance of $-50 \mathrm{~mm}$ from the leading edge. From there on the model increasingly over-predicts the position of the center of mass.

Figure 12 shows the comparison between the model prediction and the experimental data for Case 5: Run 072611-15B.9 droplet 2, droplet radius $685 \mu \mathrm{m}$, airfoil chord $0.710 \mathrm{~m}$ and airfoil velocity $90 \mathrm{~m} / \mathrm{sec}$. The droplet is the largest of the five cases to which the model was applied. The model over-predicts the position of the center of mass of the droplet over the entire range and the prediction worsens as the droplet approaches breakup. The over-prediction is 13 percent initially and is 75 percent at the breakup location.

From the results it can be seen that the model does relatively well predicting the displacement of the center of mass for the small and medium size droplets. As the droplet radius increases the model prediction deviates more from the experimental data, and for the largest droplets the model greatly overpredicts the position of the center of mass. The fact that the model prediction deviates gradually from the experimental data as the droplet size increases seems to indicate that one of the explicit or implicit assumptions may not apply at larger droplet sizes.

The assumption that the projected area of the droplet remains constant leads to under prediction of the area as the droplet deforms. This causes the model to under predict the pressure force and the displacement of the center of mass.

The assumption that the average pressure over the half droplet can be approximated by the pressure at the stagnation point is a reasonable approximation as long as the shape of the droplet does not deviate significantly from the shape of a sphere. For a droplet distorted into an asymmetric oblate spheroid shape, no experimental data was found to help determine the soundness of the average pressure approximation. The approximation did not cause a problem for the predictions of the model for small and medium droplets.

The assumption about the deformed droplet being an oblate spheroid breaks down as the droplet approaches initiation of breakup. This can be seen for the droplet in Figure 3 after frame number 100. This approximation did not affect the prediction of the displacement of the center of mass for the small and medium droplets.

Large droplets show large oscillations after being released from the droplet generator until the slip velocity begins to deform the droplet into an oblate spheroid. The initial oscillation of the droplet did not change the initial condition for the velocity of the center of mass as expressed in Equation (30). This was confirmed by studying the graphs of the major semi-axis against time for the cases studied.

Large droplets begin deforming closer to the airfoil and the time lapse from the initiation of deformation to the beginning of the breakup is shorter than the small and medium droplets but the difference is not significant enough to question the quasi-steady assumption. During deformation the large droplets follow the same pattern as the smaller ones.

The force of gravity was included in the model to improve the prediction. The following non-dimensional gravitational term was added to the right hand side of Equation (28):

$$
-\frac{K \cdot R}{U^{2}{ }_{r e l}} g
$$

where $K$ is defined in Equation (26), $R$ is the radius of the droplet, $U_{r e l}$ is the slip velocity and $g$ is the acceleration of gravity. The effect of gravity was too small to make a difference in the model prediction. 
The results indicate that the DDB model can be used for small and medium droplets. The model will be used in the analysis of the data from previous droplet breakup experiments and in the scaling of the results to large airfoils.

\section{Conclusions}

The Droplet Deformation Breakup (DDB) model developed by Ibrahim, Yang and Przekwas (Ref. 6) is used to predict the deformation of droplets approaching the leading edge of an airfoil along the stagnation line. The experimental data used in the evaluation of the model came from experiments conducted in a rotating arm facility (Refs. 1 to 3). The following conclusions can be stated about the results:

- For small and medium size droplets (radius between 200 and $500 \mu \mathrm{m}$ ) the model prediction agrees with the experimental data.

- For large droplets (radius larger than $500 \mu \mathrm{m}$ ) the model over-predicts the displacement of the center of mass by a large margin.

- The increasing deviation between the model prediction and the experimental data as droplet size increases seems to indicate that one or more of the model assumptions are invalid for large droplet sizes.

- The quasi-steady assumption needed to apply the DDB model works well for small and medium size droplets.

- The model can be used in the analysis of the deformation of small and medium droplets from previous experiments.

\section{References}

1. Vargas, M. and Feo, A., "Experimental Observations on the Deformation and Breakup of Water Droplets Near the Leading Edge of an Airfoil,” NASA/TM-2011-216946, AIAA Paper 2010-7670, August 2010.

2. Feo, A., Vargas, M. and Sor, S., "Rotating Rig Development for Droplet Deformation/Breakup and Impact Induced by Aerodynamic Surfaces,” SAE paper number 2011-38-0087, 2011.

3. Vargas, M., Sor, S. and García Magariño, A., "Mechanism of Water Droplet Breakup Near the Leading Edge of an Airfoil,” AIAA publication, presented at the 4th AIAA Atmospheric and Space Environments Conference, New Orleans, June 25-28, 2012, to be released July 2012.

4. O’Rourke, P.J. and Amsden, A.A., “The TAB Method for Numerical Calculation of Spray Droplet Breakup,” SAE paper number 872089, 1987.

5. Reitz, R.D., "Modeling Atomization Processes in High-Pressure Vaporizing Sprays,” Atomization and Spray Technology 3, 1987, pp. 309-337.

6. Ibrahim, E.A., Yang, H.Q. and Przekwas, A.J., "Modeling of Spray Droplets Deformation and Breakup,” AIAA Journal of Propulsion and Power, vol. 9, no. 4, 1993, pp. 652-654.

7. Taylor, G.I., "The Instability of Liquid Surfaces When Accelerated in a Direction Perpendicular to Their Planes,” in Batchelor, G.K. (ed) “The Scientific Papers of Sir Geoffrey Ingram Taylor,” vol. 3, Cambridge University Press, 1963, pp. 532-536. 
TABLE 1.-DATA FOR DROPLETS USED TO TEST THE DDB MODEL [Airfoil DBKUP 001—Chord size $0.710 \mathrm{~m}$.]

\begin{tabular}{|l|c|c|c|c|}
\hline Run number & $\begin{array}{c}\text { Droplet } \\
\text { number }\end{array}$ & $\begin{array}{c}\text { Airfoil } \\
\text { velocity, } \\
\mathrm{m} / \mathrm{sec}\end{array}$ & $\begin{array}{c}\text { Radius, } \\
\mu \mathrm{m}\end{array}$ & $\begin{array}{c}\text { Time at which } \\
\text { breakup begins, } \\
\mu \mathrm{sec}\end{array}$ \\
\hline 072211.1 .8 & 4 & 50 & 199 & ------ \\
\hline 072611.18 .6 & 1 & 90 & 287 & 2200 \\
\hline 072611.16 .10 & 2 & 90 & 439 & 2300 \\
\hline $072011.14 . B .1$ & 1 & 90 & 516 & 2600 \\
\hline 072611.15.B.9 & 2 & 90 & 685 & 1600 \\
\hline
\end{tabular}

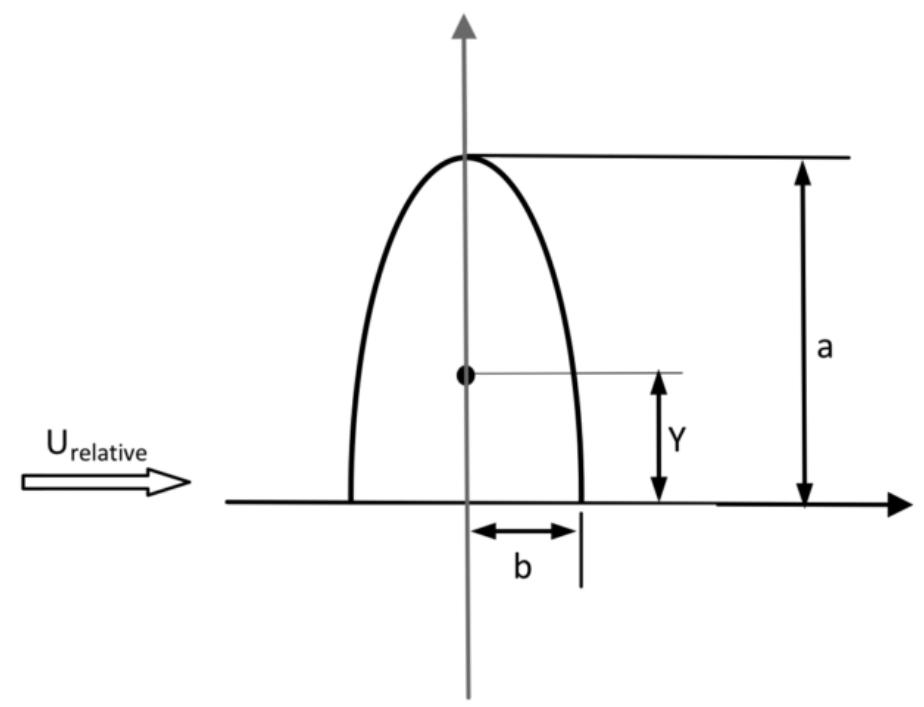

Figure 1.-Diagram of deforming half droplet. The center of mass and the axis of the oblate spheroid are indicated.

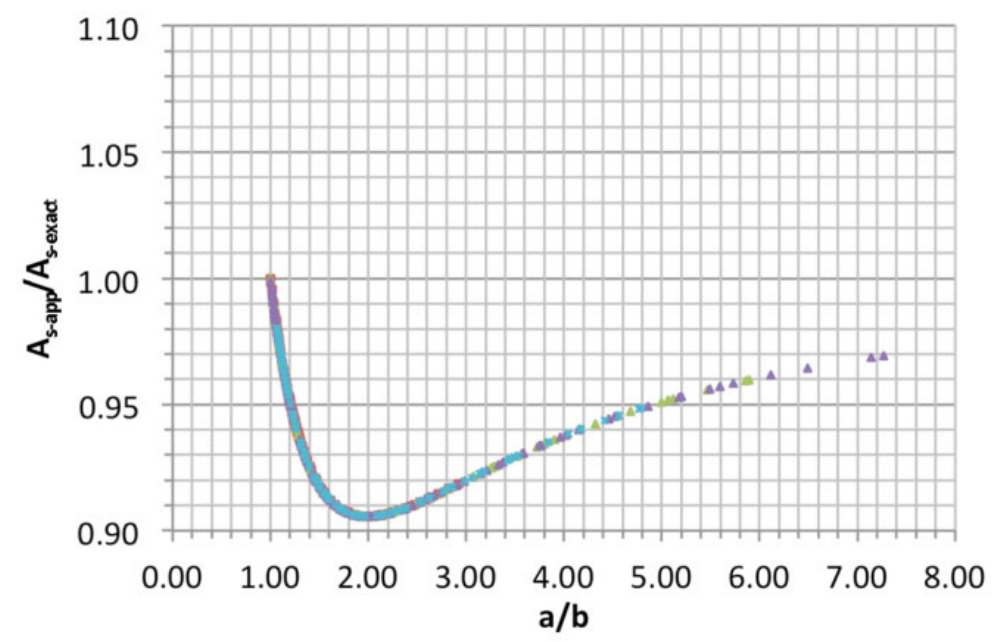

Figure 2.-Ratio of the surface area approximated by $A_{s-a p p}=2 \pi\left(a^{2}+b^{2}\right)$ over the exact value $A_{s \text {-exact }}=2 \pi\left(a^{2}+b^{2} \cdot \phi\right)$ plotted against the ratio of the large semi-axis a over the small semi-axis $b$, for the droplets: 072211 Run 1.8 Dp\#4 R = $199 \mu \mathrm{m}, 072611$ Run 18.6 Dp\#1 R = $287 \mu \mathrm{m}, 072611$ Run 16.10 Dp\#2 R = $439 \mu \mathrm{m}, 072011$ Run 14B.1 Dp\#1 R = $516 \mu \mathrm{m}, 072611$ Run 15B.9 Dp\#2 R = $685 \mu \mathrm{m}$. 


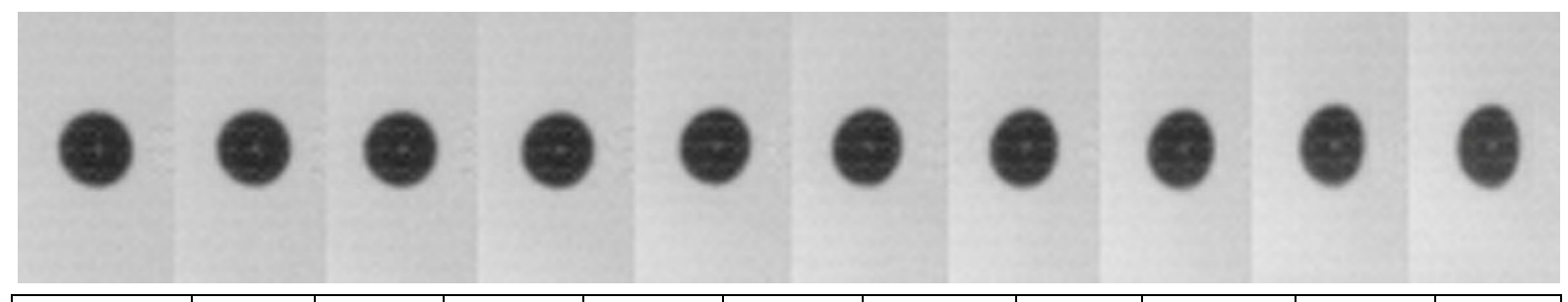

\begin{tabular}{|l|c|c|c|c|c|c|c|c|c|c|}
\hline Frame \# & 1 & 10 & 20 & 30 & 40 & 50 & 60 & 70 & 80 & 90 \\
\hline $\begin{array}{l}\text { Time } \\
(\mu \mathrm{sec})\end{array}$ & 0 & 120 & 253 & 387 & 520 & 653 & 787 & 920 & 1053 & 1187 \\
\hline $\begin{array}{l}\mathrm{x}-\mathrm{Distance} \\
(\mathrm{mm})\end{array}$ & -238.6 & -227.9 & -215.9 & -204.0 & -192.1 & -180.1 & -168.2 & -156.3 & -144.3 & -132.4 \\
\hline $\begin{array}{l}U_{\text {rel }}(\mathrm{m} / \mathrm{sec}) \\
\text { We }\end{array}$ & 14.5 & 15.3 & 16.2 & 17.3 & 18.4 & 19.6 & 20.9 & 22.3 & 23.8 & 25.5 \\
\hline $\mathrm{Re}$ & 1019 & 1075 & 1142 & 1213 & 1291 & 1375 & 1466 & 1565 & 1673 & 1792 \\
\hline Bo & 1.62 & 1.68 & 1.74 & 1.81 & 1.88 & 1.97 & 2.08 & 2.22 & 2.41 & 2.69 \\
\hline $\mathrm{Cd}$ & 0.60 & 0.56 & 0.51 & 0.47 & 0.44 & 0.40 & 0.37 & 0.35 & 0.33 & 0.32 \\
\hline
\end{tabular}

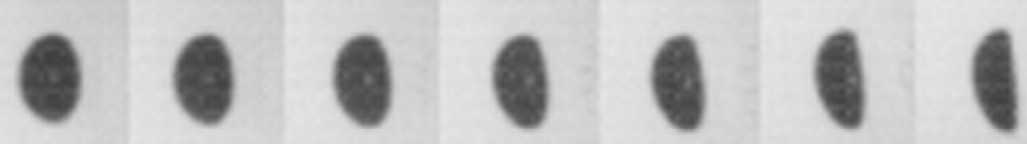

\begin{tabular}{|l|c|c|c|c|c|c|c|c|c|c|}
\hline Frame \# & 100 & 110 & 120 & 130 & 140 & 150 & 160 & 170 & 180 & 190 \\
\hline $\begin{array}{l}\text { Time } \\
(\mu \mathrm{sec})\end{array}$ & 1320 & 1453 & 1587 & 1720 & 1853 & 1987 & 2120 & 2253 & 2387 & 2520 \\
\hline $\begin{array}{l}\mathrm{x}-\mathrm{Distance} \\
(\mathrm{mm})\end{array}$ & -120.5 & -108.6 & -96.7 & -84.8 & -72.9 & -61.0 & -49.1 & -37.3 & -25.5 & -13.8 \\
\hline $\begin{array}{l}U_{\text {rel }} \\
(\mathrm{m} / \mathrm{sec})\end{array}$ & 27.4 & 29.5 & 31.9 & 34.7 & 38.0 & 41.9 & 46.7 & 52.7 & 60.3 & 70.0 \\
\hline We & 12.8 & 14.9 & 17.4 & 20.6 & 24.6 & 30.0 & 37.2 & 47.4 & 62.0 & 83.7 \\
\hline Re & 1925 & 2073 & 2242 & 2438 & 2668 & 2944 & 3281 & 3702 & 4235 & 4921 \\
\hline Bo & 3.13 & 3.82 & 4.97 & 6.89 & 10.13 & 15.66 & 25.11 & 41.32 & 69.16 & 117.05 \\
\hline Cd & 0.33 & 0.34 & 0.38 & 0.45 & 0.55 & 0.70 & 0.90 & 1.16 & 1.49 & 1.86 \\
\hline
\end{tabular}
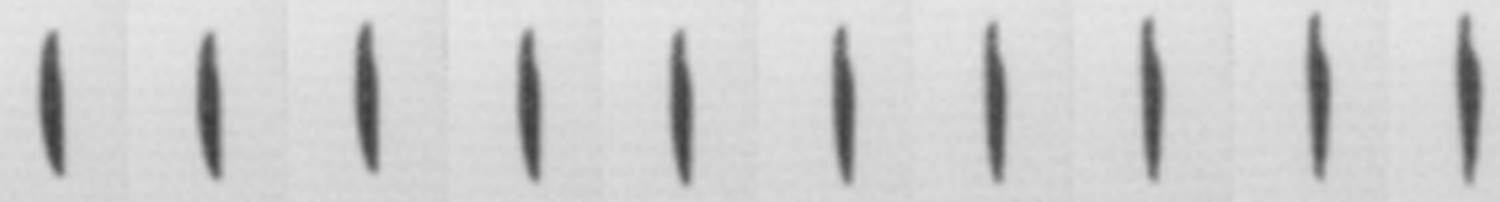

\begin{tabular}{|l|c|c|c|c|c|c|c|c|c|c|}
\hline Frame \# & 191 & 192 & 193 & 194 & 195 & 196 & 197 & 198 & 199 & 200 \\
\hline $\begin{array}{l}\text { Time } \\
(\mu s e c)\end{array}$ & 2533 & 2547 & 2560 & 2573 & 2587 & 2600 & 2612 & 2627 & 2640 & 2653 \\
\hline $\begin{array}{l}\text { x-Distance } \\
(\mathrm{mm})\end{array}$ & -12.6 & -11.5 & -10.3 & -9.2 & -8.0 & -6.8 & -5.7 & -4.5 & -3.4 & -2.3 \\
\hline $\begin{array}{l}U_{\text {rel }}(\mathrm{m} / \mathrm{sec}) \\
\text { We }\end{array}$ & 71.1 & 72.3 & 73.5 & 74.7 & 75.9 & 77.2 & 78.5 & 79.8 & 81.2 & 82.6 \\
\hline Re & 46.4 & 89.2 & 92.2 & 95.2 & 98.4 & 101.7 & 105.2 & 108.8 & 112.6 & 116.4 \\
\hline Bo & 123.42 & 130.15 & 137.26 & 144.76 & 152.68 & 161.04 & 169.87 & 179.19 & 189.03 & 199.42 \\
\hline Cd & 1.90 & 1.94 & 1.99 & 2.03 & 2.07 & 2.11 & 2.15 & 2.20 & 2.24 & 2.28 \\
\hline
\end{tabular}

Figure 3.-Sequence of deformation and initiation of breakup for Run 072011.14B.1 droplet \#1, droplet radius = $516 \mu \mathrm{m}$, airfoil chord $=0.710 \mathrm{~m}$, airfoil velocity $=90 \mathrm{~m} / \mathrm{sec}$. The table below the sequence gives the corresponding values for several of the parameters at each stage in the droplet deformation. 


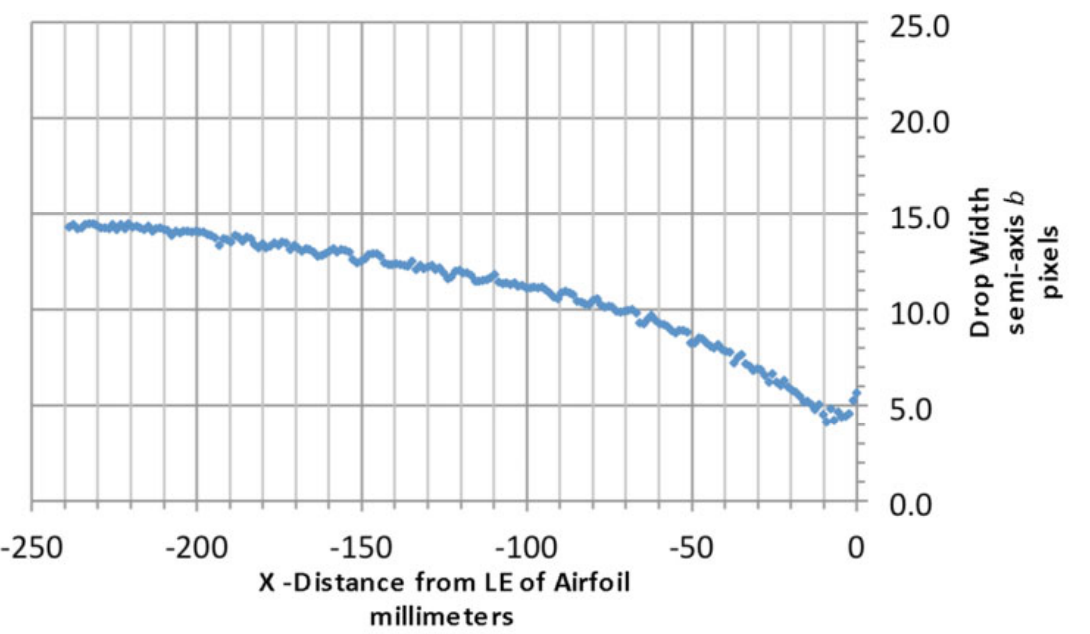

Figure 4.-Droplet width (semi-axis $b$ ) in pixels against distance from the leading edge of the airfoil in millimeters. Run 072011.14B.1 droplet \#1, droplet radius $=516 \mu \mathrm{m}$, airfoil chord $=0.710 \mathrm{~m}$, airfoil velocity $=$ $90 \mathrm{~m} / \mathrm{sec}$.

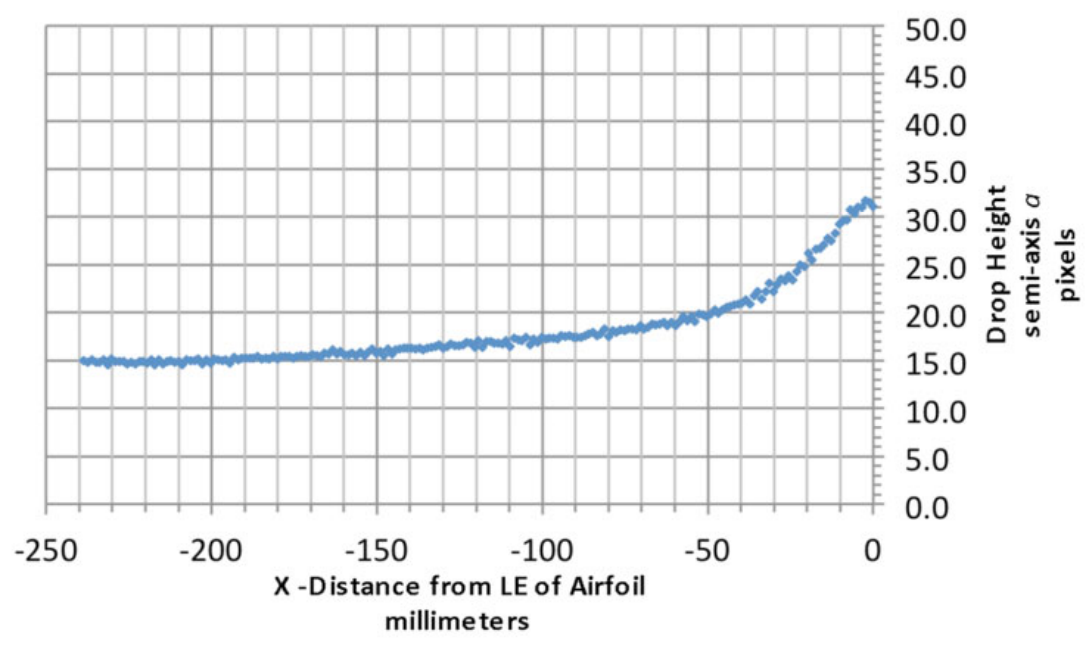

Figure 5.-Droplet Height (semi-axis a) in pixels against distance from the leading edge of the airfoil in millimeters. Run 072011.14B.1 droplet \#1, droplet radius $=516 \mu \mathrm{m}$, airfoil chord $=0.710 \mathrm{~m}$, airfoil velocity $=$ $90 \mathrm{~m} / \mathrm{sec}$. 


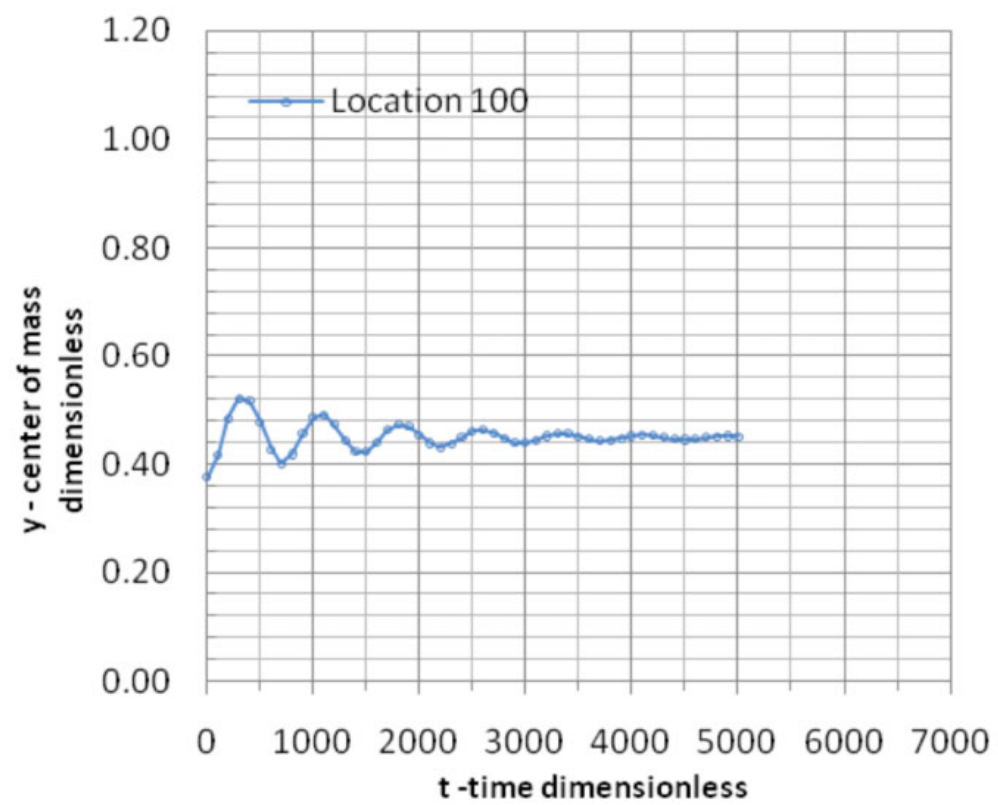

Figure 6.-Center of mass oscillation when the DDB model is solved at location 100, distance from the leading edge of the airfoil $=-120.5 \mathrm{~mm}$, Run 072011 14B.1 droplet $\# 1$, droplet radius $=516 \mu \mathrm{m}$, airfoil chord = $0.710 \mathrm{~m}$, airfoil velocity $=90 \mathrm{~m} / \mathrm{sec}$.

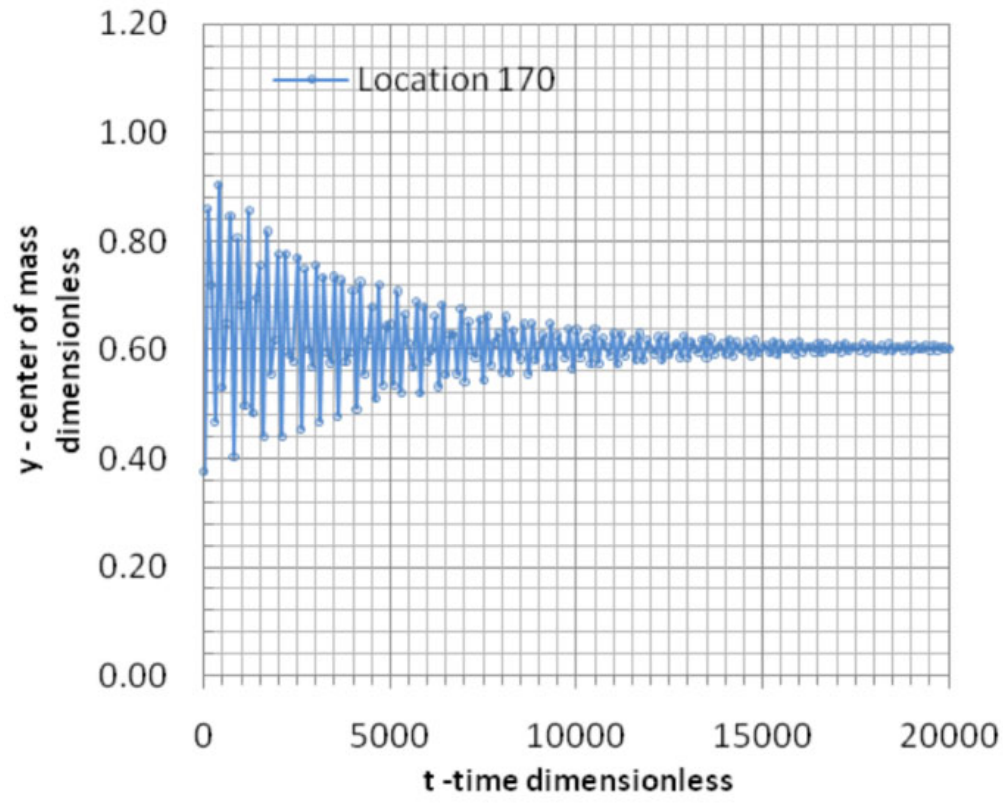

Figure 7.-Center of mass oscillation when the DDB model is solved at location 170, distance from the leading edge of the airfoil $=-37.3 \mathrm{~mm}$, Run 072011 14B.1 droplet \#1, droplet radius $=516 \mu \mathrm{m}$, airfoil chord = $0.710 \mathrm{~m}$, airfoil velocity $=90 \mathrm{~m} / \mathrm{sec}$. 


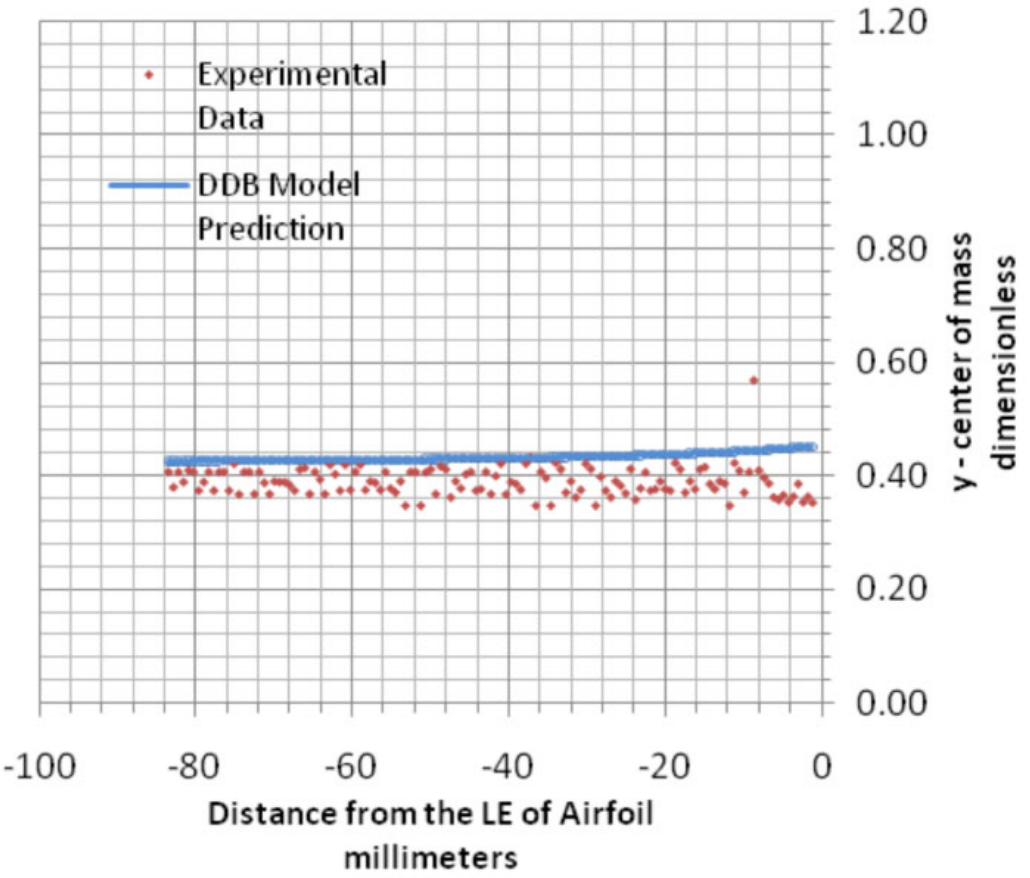

Figure 8.-DDB Model prediction compared to the experimental data for Run 0722111.8 droplet \#4, droplet radius $=199 \mu \mathrm{m}$, airfoil chord $=$ $0.710 \mathrm{~m}$, airfoil velocity $=50 \mathrm{~m} / \mathrm{sec}$.

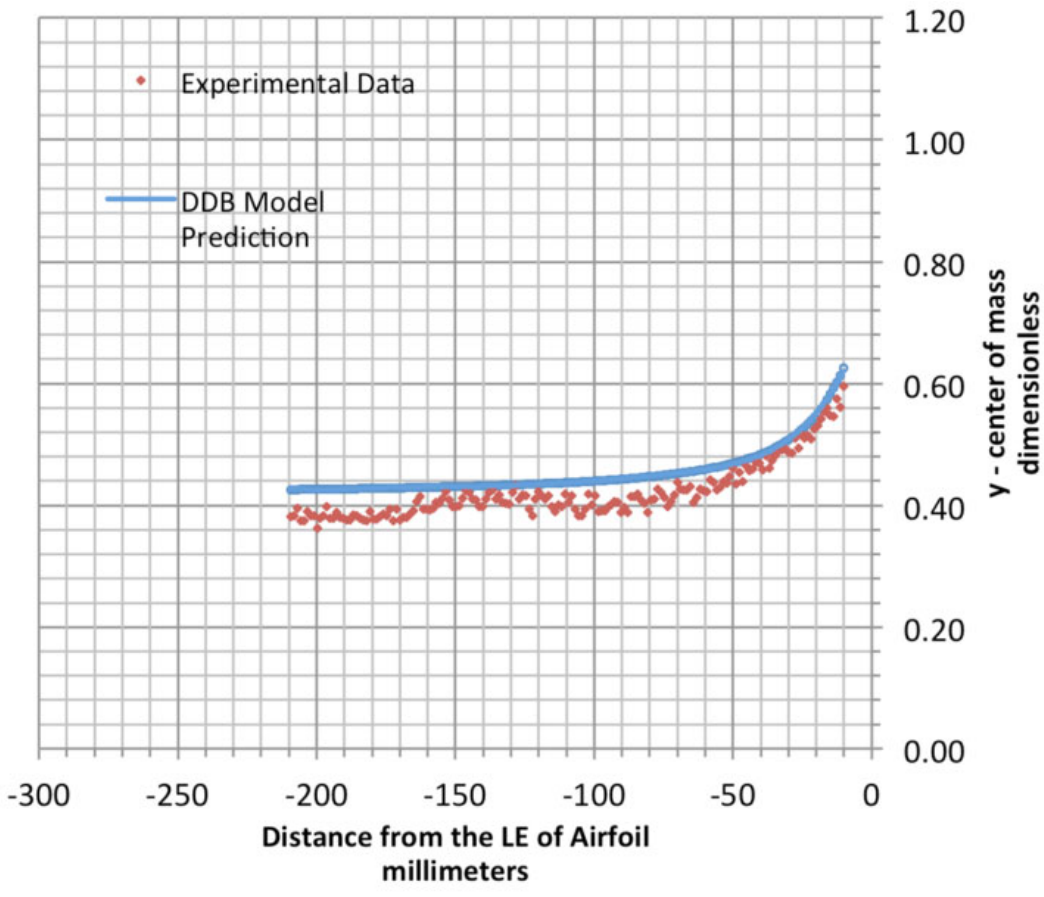

Figure 9.-DDB Model prediction compared to the experimental data for Run 07261118.6 droplet \#1, droplet radius $=287 \mu \mathrm{m}$, airfoil chord = $0.710 \mathrm{~m}$, airfoil velocity $=90 \mathrm{~m} / \mathrm{sec}$. 


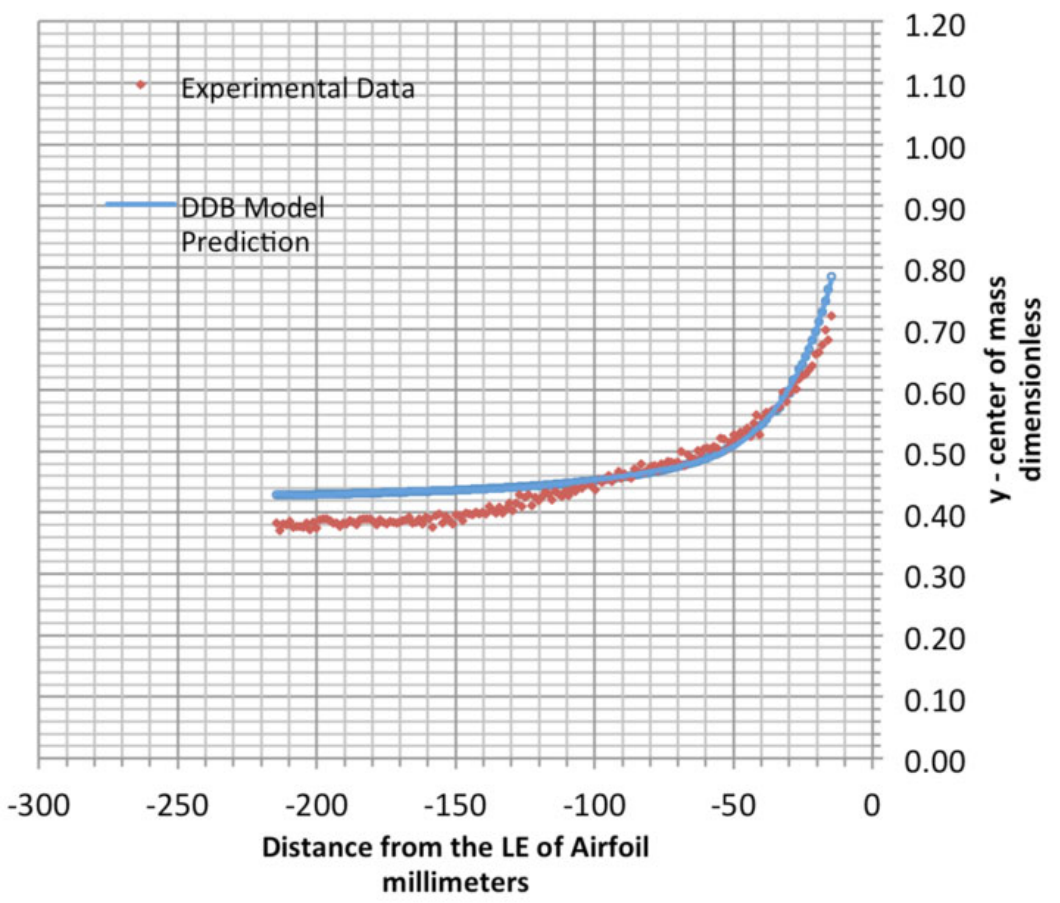

Figure 10.-DDB model prediction compared to the experimental data for Run 07261116.10 droplet \#2, droplet radius $=439 \mu \mathrm{m}$, airfoil chord $=$ $0.710 \mathrm{~m}$, airfoil velocity $=90 \mathrm{~m} / \mathrm{sec}$.

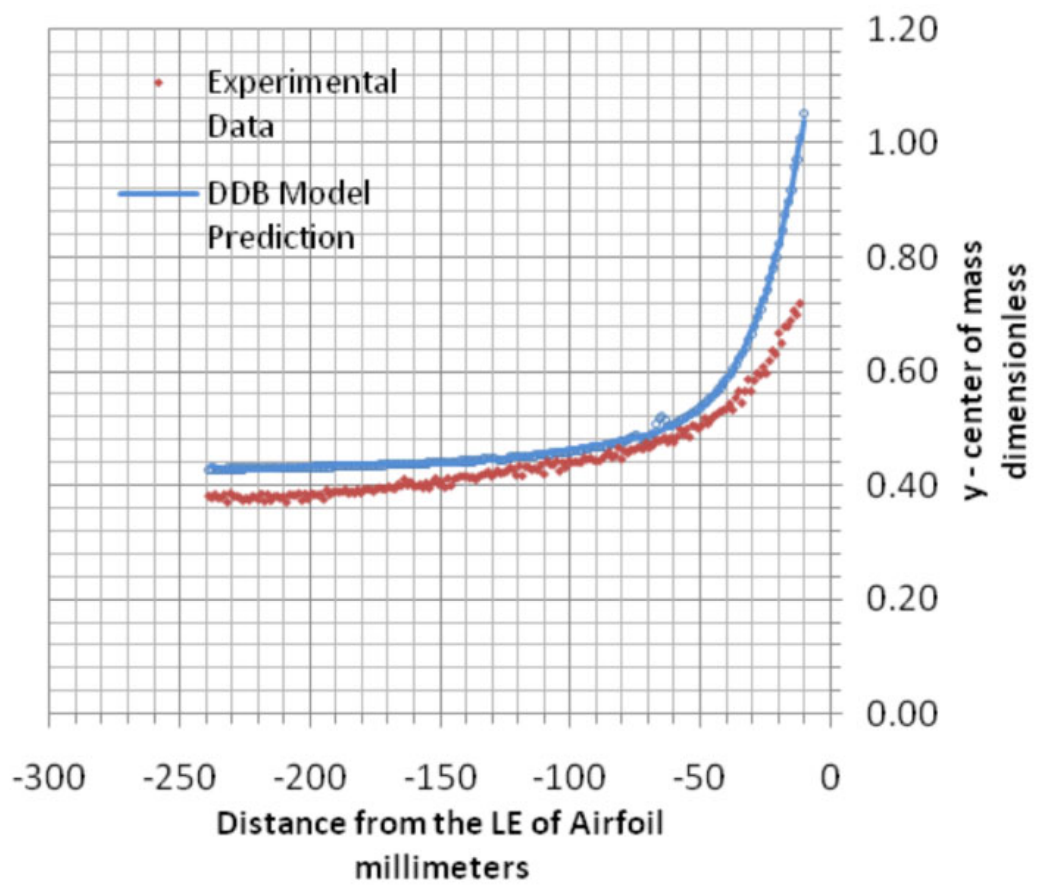

Figure 11.-DDB Model prediction compared to the experimental data for Run 072011 14B.1 droplet \#1, droplet radius $=516 \mu \mathrm{m}$, airfoil chord $=$ $0.710 \mathrm{~m}$, airfoil velocity $=90 \mathrm{~m} / \mathrm{sec}$. 


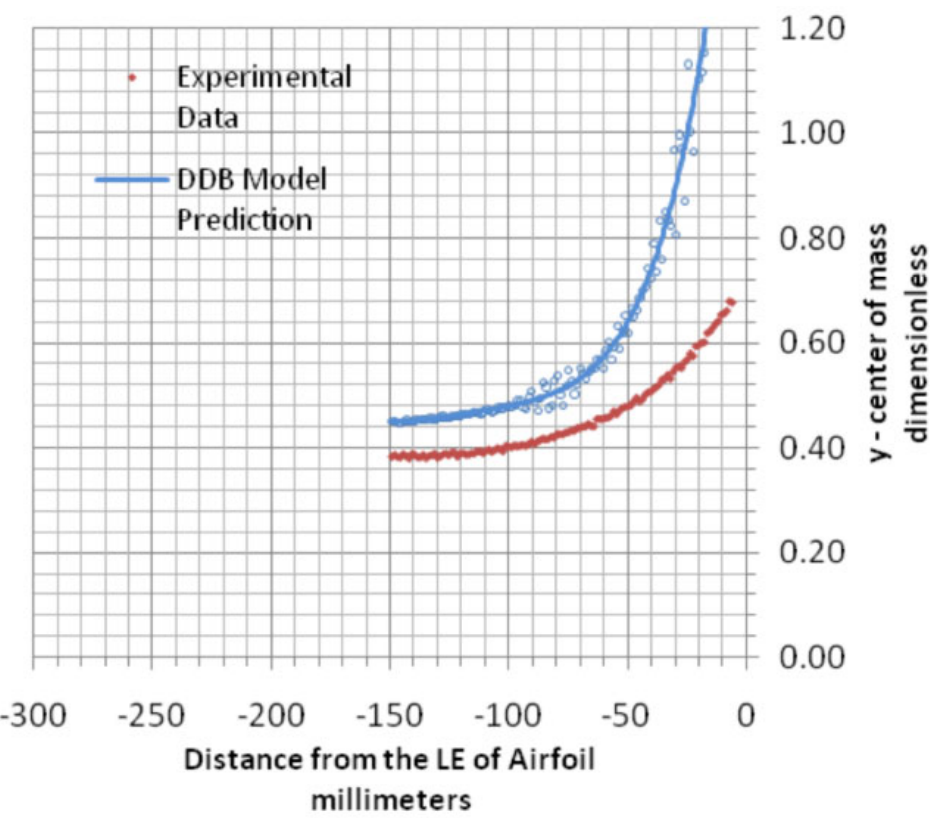

Figure 12.-DDB Model prediction compared to the experimental data for Run 072611 15B.9 droplet \#2, droplet radius $=685 \mu \mathrm{m}$, airfoil chord $=0.710 \mathrm{~m}$, airfoil velocity $=90 \mathrm{~m} / \mathrm{sec}$. 


\begin{tabular}{|c|c|c|}
\hline \multicolumn{2}{|c|}{ REPORT DOCUMENTATION PAGE } & $\begin{array}{l}\text { Form Approved } \\
\text { OMB No. 0704-0188 }\end{array}$ \\
\hline \multicolumn{3}{|c|}{ 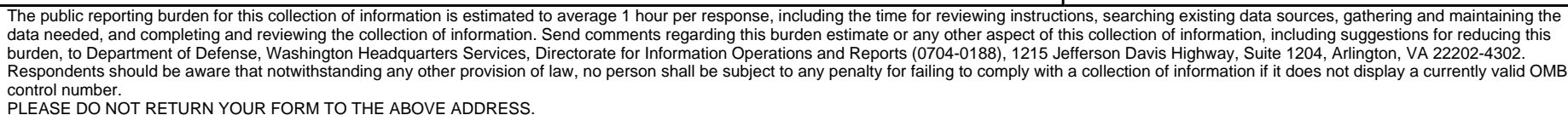 } \\
\hline $\begin{array}{l}\text { 1. REPORT DATE (DD-MM-YYYY) } \\
01-09-2012\end{array}$ & $\begin{array}{l}\text { 2. REPORT TYPE } \\
\text { Technical Memorandum }\end{array}$ & 3. DATES COVERED (From - To) \\
\hline \multirow{3}{*}{\multicolumn{2}{|c|}{$\begin{array}{l}\text { 4. TITLE AND SUBTITLE } \\
\text { Droplet Deformation Prediction With the Droplet Deformation and Breakup Model (DDB) }\end{array}$}} & 5a. CONTRACT NUMBER \\
\hline & & 5b. GRANT NUMBER \\
\hline & & 5c. PROGRAM ELEMENT NUMBER \\
\hline \multirow{3}{*}{\multicolumn{2}{|c|}{$\begin{array}{l}\text { 6. AUTHOR(S) } \\
\text { Vargas, Mario }\end{array}$}} & 5d. PROJECT NUMBER \\
\hline & & 5e. TASK NUMBER \\
\hline & & $\begin{array}{l}\text { 5f. WORK UNIT NUMBER } \\
\text { WBS 648987.02.02.03.10 }\end{array}$ \\
\hline \multicolumn{2}{|c|}{$\begin{array}{l}\text { 7. PERFORMING ORGANIZATION NAME(S) AND ADDRESS(ES) } \\
\text { National Aeronautics and Space Administration } \\
\text { John H. Glenn Research Center at Lewis Field } \\
\text { Cleveland, Ohio 44135-3191 }\end{array}$} & $\begin{array}{l}\text { 8. PERFORMING ORGANIZATION } \\
\text { REPORT NUMBER } \\
\text { E-18392 }\end{array}$ \\
\hline \multirow{2}{*}{\multicolumn{2}{|c|}{$\begin{array}{l}\text { 9. SPONSORING/MONITORING AGENCY NAME(S) AND ADDRESS(ES) } \\
\text { National Aeronautics and Space Administration } \\
\text { Washington, DC 20546-0001 }\end{array}$}} & $\begin{array}{l}\text { 10. SPONSORING/MONITOR'S } \\
\text { ACRONYM(S) } \\
\text { NASA }\end{array}$ \\
\hline & & $\begin{array}{l}\text { 11. SPONSORING/MONITORING } \\
\text { REPORT NUMBER } \\
\text { NASA/TM-2012-217705 }\end{array}$ \\
\hline \multicolumn{3}{|c|}{$\begin{array}{l}\text { 12. DISTRIBUTION/AVAILABILITY STATEMENT } \\
\text { Unclassified-Unlimited } \\
\text { Subject Category: } 03 \\
\text { Available electronically at http://www.sti.nasa.gov } \\
\text { This publication is available from the NASA Center for AeroSpace Information, 443-757-5802 }\end{array}$} \\
\hline
\end{tabular}

\section{SUPPLEMENTARY NOTES}

\section{ABSTRACT}

The Droplet Deformation and Breakup Model was used to predict deformation of droplets approaching the leading edge stagnation line of an airfoil. The quasi-steady model was solved for each position along the droplet path. A program was developed to solve the non-linear, second order, ordinary differential equation that governs the model. A fourth order Runge-Kutta method was used to solve the equation. Experimental slip velocities from droplet breakup studies were used as input to the model which required slip velocity along the particle path. The center of mass displacement predictions were compared to the experimental measurements from the droplet breakup studies for droplets with radii in the range of 200 to $700 \mathrm{~mm}$ approaching the airfoil at 50 and $90 \mathrm{~m} / \mathrm{sec}$. The model predictions were good for the displacement of the center of mass for small and medium sized droplets. For larger droplets the model predictions did not agree with the experimental results.

\section{SUBJECT TERMS}

Icing; Engine icing

\begin{tabular}{|c|c|c|c|c|}
\hline \multicolumn{3}{|c|}{ 16. SECURITY CLASSIFICATION OF: } & \multirow{2}{*}{$\begin{array}{l}\text { 17. LIMITATION OF } \\
\text { ABSTRACT } \\
\text { UU }\end{array}$} & \multirow{2}{*}{$\begin{array}{l}\text { 18. NUMBER } \\
\text { OF } \\
\text { PAGES } \\
24\end{array}$} \\
\hline $\begin{array}{l}\text { a. REPORT } \\
U\end{array}$ & $\begin{array}{l}\text { b. ABSTRACT } \\
U\end{array}$ & $\begin{array}{l}\text { c. THIS } \\
\text { PAGE } \\
\text { U }\end{array}$ & & \\
\hline
\end{tabular}



